\title{
Morphometric differences of Spirlin, Alburnoides Bipunctatus Bloch 1782 from the Major Catchements of Transylvania
}

\author{
Tudor PĂPUC ${ }^{1}$, Daniel COCAN ${ }^{1}$, Călin LAȚIU ${ }^{1}$, Paul UIUIU ${ }^{1}$, Vioara MIREȘAN ${ }^{1 *}$ \\ ${ }^{1}$ Faculty of Animal Science and Biotechnologies, University of Agricultural Sciences and Veterinary \\ Medicine Cluj-Napoca, 3-5 Mănăştur Street, 400372, Romania \\ "Corresponding author, e-mail: vmiresan@yahoo.com; vioara.miresan@usamvcluj.ro
}

Bulletin UASVM Animal Science and Biotechnologies 74(2)/ 2017

Print ISSN 1843-5262; Electronic ISSN 1843-536X

DOI:10.15835/buasvmcn-asb: 0030

\begin{abstract}
Spirlin (Alburnoides bipunctatus) is found in great numbers in most Transylvanian rivers. The species has an important role in the ecosystems in which it is found in terms of feeding and habitat occupation. The morphmetric characthers of this species vary between different habitats, outlining morphometric plasticity. Morphometric profiles were made for different populations of Spirlin, to evaluate differences in body shape. Considering that different environmental conditions influence the morphology of Spirlin populations, morphometric characteristics were determined. In order to assess differences in spirlin body shape, specimens from the four major Transylvanian catchments were collected by angling techniques. The specimens were preserved and transported to the University of Agricultural Sciences and Veterinary Medicine, Cluj-Napoca, where morphometric measurements and weight determinations were conducted in the Physiology of Aquatic Organisms Laboratory. Spirlin from Mures, Somes, Cris and Viseu catchments were studied, as it follows: 21 specimens from Aries River (Mures catchment), 61 specimens from the river Crisul Repede (Cris catchment), 21 specimens from Somesul Mic River (Somes catchment) and 20 specimens from Viseu River. A number of 39 determinations were made. The obtained results reflect the morphometric differences among populations of Spirlin from some Transylvanian main rivers.
\end{abstract}

Keywords: morphometry, Alburnoides bipunctatus, Transylvania

\section{INTRODUCTION}

The native teritorry of the spirlin (Alburnoides bipunctatus) ranges from Europe, from the Atlantic coast of France (Ladiges and Vogt, 1979), to the Middle East, in middle and upper sectors of rivers that drain in the Black, Baltic, Azov and Caspian seas (Berg, 1964). Even though the spirlin is rarely found and receives legislative protection in some countries and regions (Lusk et al., 1998), being endangered (Hensel and Mužík, 2001; Lelek, 1987), in most areas it can be found in large numbers. The microhabitat of the spirlin is usually described in general terms (Tales et al., 2004; Jurajda et al., 2007). The species has high oxygen requirements and it prefers hard substrate and low turbidity (Peňáz, 1995). These conditions make the spirlin a good indicator of water quality, being sensitive to habitat degradation (Breitenstein and Kirchhofer, 2000). The population dynamics also reflect changes in habitat (Jurajda et al., 1996). The spirlin is also an important item in the trophic chain,being consumed by most predator fish and consuming planktonic and benthic organisms (Treer et al., 2006).

Differences in environmental conditions and water constructions can produce high plasticity in morphometric characteristics (Swain et al., 1991; Azizi et al., 2015). Some authors suggest that morphometry can be related to microhabitat utilization (Kovač et al., 2006; Copp et al., 2010).

In Romania, spirlin was mentioned in some studies (Bănăduc et al., 2013; Curtean-Bănăduc 
and Bănăduc, 2007; Györe et al., 2013), but few scientific papers include detailed information about the spirlin (Bănărescu, 1964; Papadopol and Cristofor, 1980; Cocan et al., 2015). The aim of this study is to present the morphometric profile of the spirlin from four rivers from Transylvania, as well as to produce more data regarding this species in Romania.

\section{MATERIAL AND METHODS}

The studied specimens of spirlin were caught in 4 locations. 61 specimens were caught from Crisul Repede River of the Cris River Catchment, 21 specimens were collected from Somesul Mic River of the Somes River catchment, 21 specimens were collected from Aries River of the Mures River catchment and 20 specimens were caught from Viseu River catchment. The time period in which the specimens were collected spanned over 1 year, from March 2016 to the same month of the next year. The collection method consisted in angling, being a less invasive method (Bartholomew and Bohnsack, 2005; Cooke and Schramm, 2007) and a total of 123 specimens were studied. The sampling points are presented in Table 1.

The morphometric measurements could not be conducted in situ. The spirlin specimens were preserved in formalin solution $(2 \%)$ and transported to the University of Agricultural Sciences and Veterinary Medicine, Cluj-Napoca. A number of 36 measurements were conducted using an electronic scale, an electronic caliper and the Piximetre 5.9 software. The following measurements were taken: body weight (BW), body depth (BD), great perimeter (GP), small perimeter (SP), inter-orbital distance (I-oD), nostril interval $(\mathrm{N}-\mathrm{I})$, commissure interval (CI), total length (TL), standard length (SL), maximum height $(\mathrm{H})$, minimum height $(\mathrm{h})$, caudal peduncle length (CPL), head length (HL), head height (HH), snout length (SnL), upper jaw length (UjL), lower jaw length (LjL), pre-orbital distance (Pre-o-D), postorbital distance (Post-o-D), eye diameter (ED), pre-dorsal length (Pr-dL), post-dorsal length (Po-dL), pre-pectoral length (Pr-pL), post-pectoral length (Po-pL), pre-ventral length (Pr-vL), postventral length (Po-vL), pre-anal length (Pr-aL), post-anal length (Po-aL), pectoral-anal length (P$A)$, pectoral-dorsal length (P-D), pectoral-ventral length $(\mathrm{P}-\mathrm{V})$, dorsal-ventral length $(\mathrm{D}-\mathrm{V})$, dorsalanal length (D-A), ventral-anal length (V-A), dorsal fin height (DfH), pectoral fins length (PfL), ventral fins length (VfL), anal fin height (AfH), caudal fin length (CfL).

The obtained data was interpreted using the GraphPad Prism 6.07 software, rendering the mean value of a characteristic for each location, the standard error, the standard deviation, the coefficient of variation, the minimum and the maximum value. Based on these measurements, some body proportions were determined and compared. The body ratios are: head length in total length ratio (HL/TL), maximum height in total length ratio $(\mathrm{H} / \mathrm{TL})$, minimum height in total length ratio ( $h / T L)$, dorsal fin height in total length ratio (DfH/TL), snout length in head length ratio (SnL/HL), lower jaw length in upper jaw length ratio (LjL/UjL) and eye diameter in head length ratio (ED/HL).

\section{RESULTS AND DISCUSSIONS}

The obtained results from the biological material collected from Crisul Repede River (Ciucea) are presented in Table 2. Following in tables 3, 4 and 5 are presented the results from Somesul Mic River, Aries River and Viseu river respectively.

Using the above information, some body proportions were determined, as presented in Table 6. The head length in total length ratio is between 18-19\% for Somesul Mic, Aries and Viseu specimens, while it is over $19 \%$ for Crisul Repede

Tab. 1. Spirlin sampling points and GPS coordinates.

\begin{tabular}{ccccc}
\hline No. & Location & $\begin{array}{c}\text { Date of } \\
\text { sampling }\end{array}$ & $\begin{array}{c}\text { Specimens } \\
\text { caught }\end{array}$ & GPS coordinates \\
\hline $\mathbf{1}$ & Crisul Repede River (Ciucea) & 2016 March & 61 & $46^{\circ} 56^{\prime} 44.1^{\prime \prime} \mathrm{N}-22^{\circ} 49^{\prime} 03.6^{\prime \prime} \mathrm{E}$ \\
\hline $\mathbf{2}$ & Somesul Mic River (Mintiu Gherlii) & 2016 July & 21 & $47^{\circ} 03^{\prime} 55.2^{\prime \prime} \mathrm{N}-23^{\circ} 55^{\prime} 27.2^{\prime \prime} \mathrm{E}$ \\
\hline $\mathbf{3}$ & Aries River & 2016 September & 21 & $46^{\circ} 30^{\prime} 36.2^{\prime \prime} \mathrm{N}-23^{\circ} 33^{\prime} 55.2^{\prime \prime} \mathrm{E}$ \\
\hline $\mathbf{4}$ & Viseu River (Petrova) & 2017 March & 20 & $47^{\circ} 50^{\prime} 47.3^{\prime \prime} \mathrm{N}-24^{\circ} 12^{\prime} 21.6^{\prime \prime} \mathrm{E}$ \\
\hline
\end{tabular}


Tab. 2. Body measurements of spirlin (Alburnoides bipunctatus) from Crisul Repede - mean values and variability $(n=61)$

\begin{tabular}{|c|c|c|c|c|c|c|c|}
\hline Morphometric characteristics & Abbreviation & MU & $X \pm s \mathbf{s}$ & Min & Max & V\% & $\mathbf{s}$ \\
\hline Body weight & BW & $\mathrm{g}$ & $10 \pm 0.32$ & 7 & 21 & 23.87 & 2.55 \\
\hline Body depth & $\mathrm{BD}$ & $\mathrm{cm}$ & $1.12 \pm 0.01$ & 0.93 & 1.45 & 9.59 & 0.10 \\
\hline Great perimeter & GP & $\mathrm{cm}$ & $5.45 \pm 0.07$ & 4.56 & 8.14 & 10.52 & 0.57 \\
\hline Small perimeter & SP & $\mathrm{cm}$ & $2.21 \pm 0.02$ & 1.83 & 2.58 & 0.07 & 0.16 \\
\hline Inter-orbital distance & $\mathrm{I}-\mathrm{oD}$ & $\mathrm{cm}$ & $0.67 \pm 0.007$ & 0.54 & 0.83 & 8.80 & 0.05 \\
\hline Nostril interval & $\mathrm{N}-\mathrm{I}$ & $\mathrm{cm}$ & $0.39 \pm 0.005$ & 0.29 & 0.50 & 10.35 & 0.04 \\
\hline Commissure interval & $\mathrm{CI}$ & $\mathrm{cm}$ & $0.72 \pm 0.01$ & 0.53 & 0.97 & 14.45 & 0.10 \\
\hline Total length & $\mathrm{TL}$ & $\mathrm{cm}$ & $9.82 \pm 0.08$ & 8.51 & 11.89 & 6.84 & 0.67 \\
\hline Standard length & SL & $\mathrm{cm}$ & $8.24 \pm 0.07$ & 7.12 & 10.25 & 7.36 & 0.60 \\
\hline Maximum height & $\mathrm{H}$ & $\mathrm{cm}$ & $2.09 \pm 0.02$ & 1.68 & 2.80 & 9.45 & 0.19 \\
\hline Minimum height & $\mathrm{h}$ & $\mathrm{cm}$ & $0.78 \pm 0.008$ & 0.65 & 0.94 & 8.25 & 0.06 \\
\hline Caudal peduncle length & CPL & $\mathrm{cm}$ & $1.51 \pm 0.02$ & 1.21 & 1.97 & 10.64 & 0.16 \\
\hline Head length & $\mathrm{HL}$ & $\mathrm{cm}$ & $1.94 \pm 0.02$ & 1.57 & 2.42 & 9.68 & 0.18 \\
\hline Head height & $\mathrm{HH}$ & $\mathrm{cm}$ & $1.51 \pm 0.01$ & 1.29 & 1.88 & 8.58 & 0.13 \\
\hline Snout length & SnL & $\mathrm{cm}$ & $0.56 \pm 0.01$ & 0.43 & 0.89 & 14.19 & 0.08 \\
\hline Upper jaw length & UjL & $\mathrm{cm}$ & $0.50 \pm 0.01$ & 0.36 & 0.73 & 16.31 & 0.08 \\
\hline Lower jaw length & LjL & $\mathrm{cm}$ & $0.50 \pm 0.01$ & 0.35 & 0.76 & 16.76 & 0.08 \\
\hline Pre-orbital distance & Pre-o-D & $\mathrm{cm}$ & $0.45 \pm 0.01$ & 0.26 & 0.70 & 19.16 & 0.08 \\
\hline Postorbital distance & Post-o-D & $\mathrm{cm}$ & $0.87 \pm 0.009$ & 0.73 & 1.06 & 8.78 & 0.07 \\
\hline Eye diameter & ED & $\mathrm{cm}$ & $0.55 \pm 0.007$ & 0.45 & 0.77 & 10.00 & 0.05 \\
\hline Pre-dorsal length & Pr-dL & $\mathrm{cm}$ & $4.45 \pm 0.04$ & 3.75 & 5.49 & 7.64 & 0.34 \\
\hline Post-dorsal length & Po-dL & $\mathrm{cm}$ & $4.04 \pm 0.04$ & 3.46 & 5.03 & 7.90 & 0.32 \\
\hline Pre-pectoral length & Pr-pL & $\mathrm{cm}$ & $2.01 \pm 0.02$ & 1.66 & 2.49 & 9.53 & 0.19 \\
\hline Post-pectoral length & Po-pL & $\mathrm{cm}$ & $6.32 \pm 0.06$ & 5.38 & 7.98 & 8.10 & 0.51 \\
\hline Pre-ventral length & Pr-vL & $\mathrm{cm}$ & $3.94 \pm 0.04$ & 3.40 & 4.93 & 9.03 & 0.35 \\
\hline Post-ventral length & Po-vL & $\mathrm{cm}$ & $4.52 \pm 0.04$ & 3.67 & 5.74 & 7.93 & 0.35 \\
\hline Pre-anal length & Pr-aL & $\mathrm{cm}$ & $5.38 \pm 0.05$ & 4.63 & 6.70 & 7.94 & 0.42 \\
\hline Post-anal length & Po-aL & $\mathrm{cm}$ & $3.08 \pm 0.03$ & 2.53 & 3.87 & 8.01 & 0.24 \\
\hline Pectoral-anal length & P-A & $\mathrm{cm}$ & $3.40 \pm 0.04$ & 2.75 & 4.36 & 9.13 & 0.31 \\
\hline Pectoral-dorsal length & P-D & $\mathrm{cm}$ & $2.89 \pm 0.03$ & 2.33 & 3.70 & 8.97 & 0.26 \\
\hline Pectoral-ventral length & $\mathrm{P}-\mathrm{V}$ & $\mathrm{cm}$ & $1.93 \pm 0.02$ & 1.51 & 2.46 & 11.08 & 0.21 \\
\hline Dorsal-ventral length & D-V & $\mathrm{cm}$ & $2.08 \pm 0.02$ & 1.68 & 2.77 & 9.46 & 0.19 \\
\hline Dorsal-anal length & D-A & $\mathrm{cm}$ & $2.21 \pm 0.02$ & 1.84 & 2.85 & 8.28 & 0.18 \\
\hline Ventral-anal length & V-A & $\mathrm{cm}$ & $1.49 \pm 0.02$ & 1.15 & 1.92 & 11.06 & 0.16 \\
\hline Dorsal fin height & DfH & $\mathrm{cm}$ & $1.81 \pm 0.02$ & 1.48 & 2.38 & 8.73 & 0.15 \\
\hline Pectoral fins length & PfL & $\mathrm{cm}$ & $1.37 \pm 0.02$ & 1.00 & 1.78 & 13.43 & 0.18 \\
\hline Ventral fins length & VfL & $\mathrm{cm}$ & $1.19 \pm 0.01$ & 0.82 & 1.64 & 11.61 & 0.13 \\
\hline Anal fin height & AfH & $\mathrm{cm}$ & $1.34 \pm 0.01$ & 0.98 & 1.72 & 11.23 & 0.15 \\
\hline Caudal fin length & $\mathrm{Cfl}$ & $\mathrm{cm}$ & $1.77 \pm 0.02$ & 1.46 & 2.20 & 8.84 & 0.15 \\
\hline
\end{tabular}

Note: MU - measurement unit; X - mean value; sx - standard error; V\% - coefficient of variation; s - standard deviation; Min - minimum value; Max - maximum value. 
Tab. 3. Body measurements of spirlin (Alburnoides bipunctatus) from Somesul Mic - mean values and variability $(\mathrm{n}=21)$

\begin{tabular}{|c|c|c|c|c|c|c|c|}
\hline Morphometric characteristics & Abbreviation & MU & $X \pm s \mathbf{s}$ & Min & Max & V\% & $\mathbf{s}$ \\
\hline Body weight & BW & $\mathrm{g}$ & $8.85 \pm 1.02$ & 4 & 22 & 51.83 & 4.59 \\
\hline Body depth & $\mathrm{BD}$ & $\mathrm{cm}$ & $1.08 \pm 0.04$ & 0.84 & 1.51 & 19.27 & 0.20 \\
\hline Great perimeter & GP & $\mathrm{cm}$ & $4.96 \pm 0.20$ & 3.88 & 7.05 & 18.11 & 0.89 \\
\hline Small perimeter & SP & $\mathrm{cm}$ & $1.91 \pm 0.07$ & 1.48 & 2.59 & 17.79 & 0.34 \\
\hline Inter-orbital distance & I-oD & $\mathrm{cm}$ & $0.59 \pm 0.02$ & 0.44 & 0.90 & 18.28 & 0.02 \\
\hline Nostril interval & $\mathrm{N}-\mathrm{I}$ & $\mathrm{cm}$ & $0.34 \pm 0.01$ & 0.22 & 0.49 & 18.05 & 0.01 \\
\hline Commissure interval & $\mathrm{CI}$ & $\mathrm{cm}$ & $0.50 \pm 0.01$ & 0.36 & 0.72 & 17.26 & 0.08 \\
\hline Total length & $\mathrm{TL}$ & $\mathrm{cm}$ & $8.89 \pm 0.29$ & 6.81 & 12.2 & 14.95 & 1.33 \\
\hline Standard length & SL & $\mathrm{cm}$ & $7.40 \pm 0.25$ & 5.93 & 10.3 & 15.28 & 1.31 \\
\hline Maximum height & $\mathrm{H}$ & $\mathrm{cm}$ & $1.91 \pm 0.08$ & 1.47 & 2.78 & 19.04 & 0.36 \\
\hline Minimum height & $\mathrm{h}$ & $\mathrm{cm}$ & $0.69 \pm 0.02$ & 0.53 & 0.92 & 14.74 & 0.10 \\
\hline Caudal peduncle length & CPL & $\mathrm{cm}$ & $1.33 \pm 0.05$ & 0.97 & 2.01 & 18.15 & 0.24 \\
\hline Head length & HL & $\mathrm{cm}$ & $1.68 \pm 0.06$ & 1.35 & 2.52 & 16.82 & 0.28 \\
\hline Head height & $\mathrm{HH}$ & $\mathrm{cm}$ & $1.24 \pm 0.04$ & 0.94 & 1.77 & 16.29 & 0.20 \\
\hline Snout length & SnL & $\mathrm{cm}$ & $0.51 \pm 0.02$ & 0.40 & 0.84 & 21.32 & 0.11 \\
\hline Upper jaw length & UjL & $\mathrm{cm}$ & $0.52 \pm 0.01$ & 0.39 & 0.75 & 15.59 & 0.08 \\
\hline Lower jaw length & LjL & $\mathrm{cm}$ & $0.53 \pm 0.01$ & 0.43 & 0.80 & 15.66 & 0.08 \\
\hline Pre-orbital distance & Pre-o-D & $\mathrm{cm}$ & $0.39 \pm 0.02$ & 0.29 & 0.66 & 23.03 & 0.09 \\
\hline Postorbital distance & Post-o-D & $\mathrm{cm}$ & $0.77 \pm 0.03$ & 0.58 & 1.18 & 17.36 & 0.13 \\
\hline Eye diameter & ED & $\mathrm{cm}$ & $0.49 \pm 0.01$ & 0.41 & 0.71 & 14.65 & 0.07 \\
\hline Pre-dorsal length & Pr-dL & $\mathrm{cm}$ & $3.95 \pm 0.14$ & 3.15 & 5.54 & 16.30 & 0.64 \\
\hline Post-dorsal length & Po-dL & $\mathrm{cm}$ & $3.66 \pm 0.11$ & 2.95 & 4.97 & 14.57 & 0.53 \\
\hline Pre-pectoral length & Pr-pL & $\mathrm{cm}$ & $1.72 \pm 0.06$ & 1.36 & 2.48 & 15.88 & 0.27 \\
\hline Post-pectoral length & Po-pL & $\mathrm{cm}$ & $5.81 \pm 0.19$ & 4.54 & 7.99 & 15.37 & 0.89 \\
\hline Pre-ventral length & Pr-vL & $\mathrm{cm}$ & $3.44 \pm 0.13$ & 2.54 & 4.73 & 16.89 & 0.58 \\
\hline Post-ventral length & Po-vL & $\mathrm{cm}$ & $4.21 \pm 0.13$ & 3.36 & 5.89 & 14.37 & 0.60 \\
\hline Pre-anal length & Pr-aL & $\mathrm{cm}$ & $4.75 \pm 0.17$ & 3.64 & 6.51 & 16.53 & 0.78 \\
\hline Post-anal length & Po-aL & $\mathrm{cm}$ & $2.86 \pm 0.08$ & 2.34 & 4.02 & 14.05 & 0.40 \\
\hline Pectoral-anal length & $\mathrm{P}-\mathrm{A}$ & $\mathrm{cm}$ & $3.07 \pm 0.12$ & 2.26 & 4.11 & 17.54 & 0.54 \\
\hline Pectoral-dorsal length & P-D & $\mathrm{cm}$ & $2.69 \pm 0.10$ & 2.08 & 3.77 & 17.30 & 0.46 \\
\hline Pectoral-ventral length & $\mathrm{P}-\mathrm{V}$ & $\mathrm{cm}$ & $1.73 \pm 0.07$ & 1.17 & 2.37 & 19.37 & 0.33 \\
\hline Dorsal-ventral length & D-V & $\mathrm{cm}$ & $1.96 \pm 0.07$ & 1.54 & 2.86 & 17.91 & 0.35 \\
\hline Dorsal-anal length & D-A & $\mathrm{cm}$ & $1.97 \pm 0.07$ & 1.50 & 2.77 & 17.81 & 0.35 \\
\hline Ventral-anal length & $\mathrm{V}-\mathrm{A}$ & $\mathrm{cm}$ & $1.37 \pm 0.05$ & 0.98 & 1.91 & 17.63 & 0.24 \\
\hline Dorsal fin height & DfH & $\mathrm{cm}$ & $1.62 \pm 0.05$ & 1.22 & 2.27 & 15.68 & 0.25 \\
\hline Pectoral fins length & PfL & $\mathrm{cm}$ & $1.12 \pm 0.04$ & 0.78 & 1.73 & 19.66 & 0.22 \\
\hline Ventral fins length & VfL & $\mathrm{cm}$ & $1.02 \pm 0.04$ & 0.73 & 1.53 & 18.67 & 0.19 \\
\hline Anal fin height & AfH & $\mathrm{cm}$ & $1.24 \pm 0.04$ & 0.89 & 1.77 & 16.11 & 0.20 \\
\hline Caudal fin length & $\mathrm{Cfl}$ & $\mathrm{cm}$ & $1.59 \pm 0.06$ & 1.00 & 2.25 & 18.01 & 0.28 \\
\hline
\end{tabular}

Note: MU - measurement unit; X - mean value; sx - standard error; V\% - coefficient of variation; s - standard deviation; Min - minimum value; Max - maximum value. 
Tab. 4. Body measurements of spirlin (Alburnoides bipunctatus) from Aries - mean values and variability $(n=21)$

\begin{tabular}{|c|c|c|c|c|c|c|c|}
\hline $\begin{array}{l}\text { Morphometric } \\
\text { characteristics }\end{array}$ & Abbreviation & MU & $X \pm s \mathbf{s}$ & Min & Max & V\% & $\mathbf{s}$ \\
\hline Body weight & BW & $\mathrm{g}$ & $5.57 \pm 0.36$ & 3 & 9 & 29.08 & 1.62 \\
\hline Body depth & BD & $\mathrm{cm}$ & $0.91 \pm 0.01$ & 0.79 & 1.07 & 8.32 & 0.07 \\
\hline Great perimeter & GP & $\mathrm{cm}$ & $4.36 \pm 0.09$ & 3.62 & 5.22 & 9.63 & 0.42 \\
\hline Small perimeter & SP & $\mathrm{cm}$ & $1.82 \pm 0.03$ & 1.51 & 2.19 & 9.46 & 0.17 \\
\hline Inter-orbital distance & I-oD & $\mathrm{cm}$ & $0.55 \pm 0.01$ & 0.49 & 0.67 & 8.59 & 0.04 \\
\hline Nostril interval & N-I & $\mathrm{cm}$ & $0.30 \pm 0.006$ & 0.25 & 0.35 & 9.04 & 0.02 \\
\hline Commissure interval & $\mathrm{CI}$ & $\mathrm{cm}$ & $0.42 \pm 0.008$ & 0.36 & 0.50 & 9.14 & 0.03 \\
\hline Total length & $\mathrm{TL}$ & $\mathrm{cm}$ & $8.76 \pm 0.15$ & 7.62 & 10.3 & 7.68 & 0.67 \\
\hline Standard length & SL & $\mathrm{cm}$ & $7.13 \pm 0.12$ & 6.22 & 8.41 & 7.76 & 0.55 \\
\hline Maximum height & $\mathrm{H}$ & $\mathrm{cm}$ & $1.71 \pm 0.03$ & 1.39 & 2.01 & 10.15 & 0.17 \\
\hline Minimum height & $\mathrm{h}$ & $\mathrm{cm}$ & $0.66 \pm 0.01$ & 0.57 & 0.77 & 8.18 & 0.05 \\
\hline Caudal peduncle length & CPL & $\mathrm{cm}$ & $1.36 \pm 0.02$ & 1.18 & 1.55 & 7.34 & 0.10 \\
\hline Head length & HL & $\mathrm{cm}$ & $1.59 \pm 0.02$ & 1.43 & 1.83 & 7.44 & 0.11 \\
\hline Head height & $\mathrm{HH}$ & $\mathrm{cm}$ & $1.18 \pm 0.02$ & 1.04 & 1.36 & 8.07 & 0.09 \\
\hline Snout length & SnL & $\mathrm{cm}$ & $0.46 \pm 0.01$ & 0.39 & 0.61 & 12.43 & 0.05 \\
\hline Upper jaw length & UjL & $\mathrm{cm}$ & $0.52 \pm 0.01$ & 0.40 & 0.63 & 11.03 & 0.05 \\
\hline Lower jaw length & LjL & $\mathrm{cm}$ & $0.55 \pm 0.01$ & 0.45 & 0.65 & 9.48 & 0.05 \\
\hline Pre-orbital distance & Pre-o-D & $\mathrm{cm}$ & $0.35 \pm 0.01$ & 0.30 & 0.45 & 13.06 & 0.04 \\
\hline Postorbital distance & Post-o-D & $\mathrm{cm}$ & $0.72 \pm 0.01$ & 0.65 & 0.81 & 6.85 & 0.04 \\
\hline Eye diameter & ED & $\mathrm{cm}$ & $0.51 \pm 0.007$ & 0.47 & 0.59 & 6.36 & 0.03 \\
\hline Pre-dorsal length & Pr-dL & $\mathrm{cm}$ & $3.72 \pm 0.06$ & 3.39 & 4.28 & 7.17 & 0.26 \\
\hline Post-dorsal length & Po-dL & $\mathrm{cm}$ & $3.61 \pm 0.07$ & 3.19 & 4.31 & 8.16 & 0.29 \\
\hline Pre-pectoral length & Pr-pL & $\mathrm{cm}$ & $1.65 \pm 0.03$ & 1.43 & 1.89 & 7.92 & 0.13 \\
\hline Post-pectoral length & Po-pL & $\mathrm{cm}$ & $5.64 \pm 0.11$ & 5.07 & 6.77 & 8.16 & 0.46 \\
\hline Pre-ventral length & Pr-vL & $\mathrm{cm}$ & $3.29 \pm 0.06$ & 2.92 & 3.88 & 8.64 & 0.28 \\
\hline Post-ventral length & Po-vL & $\mathrm{cm}$ & $4.17 \pm 0.11$ & 3.56 & 5.45 & 10.97 & 0.45 \\
\hline Pre-anal length & Pr-aL & $\mathrm{cm}$ & $4.59 \pm 0.09$ & 4.01 & 5.43 & 8.87 & 0.40 \\
\hline Post-anal length & Po-aL & $\mathrm{cm}$ & $2.80 \pm 0.04$ & 2.51 & 3.20 & 6.48 & 0.18 \\
\hline Pectoral-anal length & P-A & $\mathrm{cm}$ & $2.97 \pm 0.07$ & 2.51 & 3.68 & 10.20 & 0.30 \\
\hline Pectoral-dorsal length & P-D & $\mathrm{cm}$ & $2.47 \pm 0.05$ & 2.18 & 2.96 & 9.02 & 0.22 \\
\hline Pectoral-ventral length & $\mathrm{P}-\mathrm{V}$ & $\mathrm{cm}$ & $1.66 \pm 0.04$ & 1.40 & 2.08 & 10.57 & 0.17 \\
\hline Dorsal-ventral length & $\mathrm{D}-\mathrm{V}$ & $\mathrm{cm}$ & $1.73 \pm 0.04$ & 1.50 & 2.02 & 9.82 & 0.17 \\
\hline Dorsal-anal length & D-A & $\mathrm{cm}$ & $1.86 \pm 0.04$ & 1.58 & 2.20 & 10.48 & 0.19 \\
\hline Ventral-anal length & V-A & $\mathrm{cm}$ & $1.32 \pm 0.04$ & 1.07 & 1.67 & 13.61 & 0.18 \\
\hline Dorsal fin height & DfH & $\mathrm{cm}$ & $1.71 \pm 0.03$ & 1.44 & 2.05 & 9.56 & 0.16 \\
\hline Pectoral fins length & PfL & $\mathrm{cm}$ & $1.30 \pm 0.03$ & 1.12 & 1.54 & 10.07 & 0.13 \\
\hline Ventral fins length & VfL & $\mathrm{cm}$ & $1.07 \pm 0.03$ & 0.83 & 1.28 & 12.75 & 0.13 \\
\hline Anal fin height & AfH & $\mathrm{cm}$ & $1.20 \pm 0.03$ & 1.02 & 1.46 & 10.33 & 0.12 \\
\hline Caudal fin length & $\mathrm{Cfl}$ & $\mathrm{cm}$ & $1.71 \pm 0.03$ & 1.48 & 1.99 & 8.47 & 0.14 \\
\hline
\end{tabular}

Note: MU - measurement unit; X - mean value; sx - standard error; V\% - coefficient of variation; s - standard deviation; Min - minimum value; Max - maximum value. 
Tab. 5. Body measurements of spirlin (Alburnoides bipunctatus) from Viseu - mean values and variability $(n=20)$

\begin{tabular}{|c|c|c|c|c|c|c|c|}
\hline Morphometric characteristics & Abbreviation & MU & $\mathbf{X} \pm \mathbf{s X}$ & Min & Max & V\% & $\mathbf{s}$ \\
\hline Body weight & BW & $\mathrm{g}$ & $5.37 \pm 0.59$ & 3.12 & 14.52 & 48.11 & 2.58 \\
\hline Body depth & $\mathrm{BD}$ & $\mathrm{cm}$ & $0.83 \pm 0.03$ & 0.64 & 1.23 & 16.55 & 0.13 \\
\hline Great perimeter & GP & $\mathrm{cm}$ & $3.98 \pm 0.16$ & 3.11 & 6.03 & 17.58 & 0.70 \\
\hline Small perimeter & SP & $\mathrm{cm}$ & $1.60 \pm 0.04$ & 1.32 & 2.20 & 13.37 & 0.21 \\
\hline Inter-orbital distance & $\mathrm{I}-\mathrm{oD}$ & $\mathrm{cm}$ & $0.49 \pm 0.01$ & 0.42 & 0.66 & 12.96 & 0.06 \\
\hline Nostril interval & $\mathrm{N}-\mathrm{I}$ & $\mathrm{cm}$ & $0.25 \pm 0.007$ & 0.18 & 0.32 & 13.22 & 0.03 \\
\hline Commissure interval & $\mathrm{CI}$ & $\mathrm{cm}$ & $0.47 \pm 0.01$ & 0.36 & 0.62 & 14.50 & 0.06 \\
\hline Total length & $\mathrm{TL}$ & $\mathrm{cm}$ & $8.08 \pm 0.22$ & 7.02 & 11.03 & 11.89 & 0.96 \\
\hline Standard length & SL & $\mathrm{cm}$ & $6.59 \pm 0.18$ & 5.71 & 9.05 & 12.32 & 0.81 \\
\hline Maximum height & $\mathrm{H}$ & $\mathrm{cm}$ & $1.55 \pm 0.05$ & 1.28 & 2.34 & 16.36 & 0.25 \\
\hline Minimum height & $\mathrm{h}$ & $\mathrm{cm}$ & $0.60 \pm 0.01$ & 0.52 & 0.85 & 12.86 & 0.07 \\
\hline Caudal peduncle length & CPL & $\mathrm{cm}$ & $1.34 \pm 0.04$ & 0.85 & 1.73 & 14.77 & 0.19 \\
\hline Head length & $\mathrm{HL}$ & $\mathrm{cm}$ & $1.52 \pm 0.04$ & 1.31 & 2.01 & 11.90 & 0.18 \\
\hline Head height & $\mathrm{HH}$ & $\mathrm{cm}$ & $1.11 \pm 0.03$ & 0.95 & 1.50 & 12.63 & 0.14 \\
\hline Snout length & SnL & $\mathrm{cm}$ & $0.51 \pm 0.01$ & 0.37 & 0.73 & 16.72 & 0.08 \\
\hline Upper jaw length & UjL & $\mathrm{cm}$ & $0.52 \pm 0.01$ & 0.42 & 0.66 & 11.69 & 0.06 \\
\hline Lower jaw length & LjL & $\mathrm{cm}$ & $0.55 \pm 0.01$ & 0.44 & 0.71 & 11.37 & 0.06 \\
\hline Pre-orbital distance & Pre-o-D & $\mathrm{cm}$ & $0.39 \pm 0.01$ & 0.29 & 0.58 & 18.27 & 0.07 \\
\hline Postorbital distance & Post-o-D & $\mathrm{cm}$ & $0.62 \pm 0.02$ & 0.53 & 0.90 & 14.81 & 0.09 \\
\hline Eye diameter & ED & $\mathrm{cm}$ & $0.47 \pm 0.009$ & 0.43 & 0.58 & 8.36 & 0.03 \\
\hline Pre-dorsal length & Pr-dL & $\mathrm{cm}$ & $3.49 \pm 0.10$ & 3.00 & 4.77 & 12.50 & 0.43 \\
\hline Post-dorsal length & Po-dL & $\mathrm{cm}$ & $3.27 \pm 0.09$ & 2.71 & 4.55 & 12.73 & 0.41 \\
\hline Pre-pectoral length & Pr-pL & $\mathrm{cm}$ & $1.57 \pm 0.03$ & 1.37 & 2.06 & 10.81 & 0.16 \\
\hline Post-pectoral length & Po-pL & $\mathrm{cm}$ & $5.10 \pm 0.15$ & 4.35 & 7.12 & 12.85 & 0.65 \\
\hline Pre-ventral length & Pr-vL & $\mathrm{cm}$ & $3.00 \pm 0.07$ & 2.60 & 3.98 & 11.55 & 0.34 \\
\hline Post-ventral length & Po-vL & $\mathrm{cm}$ & $3.74 \pm 0.11$ & 3.14 & 5.31 & 13.48 & 0.50 \\
\hline Pre-anal length & Pr-aL & $\mathrm{cm}$ & $4.16 \pm 0.11$ & 3.56 & 5.71 & 12.35 & 0.51 \\
\hline Post-anal length & Po-aL & $\mathrm{cm}$ & $2.58 \pm 0.07$ & 2.14 & 3.56 & 12.60 & 0.32 \\
\hline Pectoral-anal length & P-A & $\mathrm{cm}$ & $2.62 \pm 0.08$ & 2.16 & 3.73 & 13.63 & 0.35 \\
\hline Pectoral-dorsal length & P-D & $\mathrm{cm}$ & $2.26 \pm 0.07$ & 1.87 & 3.35 & 15.04 & 0.34 \\
\hline Pectoral-ventral length & $\mathrm{P}-\mathrm{V}$ & $\mathrm{cm}$ & $1.45 \pm 0.04$ & 1.26 & 1.96 & 12.62 & 0.18 \\
\hline Dorsal-ventral length & D-V & $\mathrm{cm}$ & $1.58 \pm 0.05$ & 1.29 & 2.40 & 16.40 & 0.26 \\
\hline Dorsal-anal length & D-A & $\mathrm{cm}$ & $1.63 \pm 0.05$ & 1.30 & 2.44 & 15.17 & 0.24 \\
\hline Ventral-anal length & $\mathrm{V}-\mathrm{A}$ & $\mathrm{cm}$ & $1.19 \pm 0.04$ & 0.95 & 1.77 & 16.00 & 0.19 \\
\hline Dorsal fin height & DfH & $\mathrm{cm}$ & $1.49 \pm 0.03$ & 1.18 & 1.96 & 11.43 & 0.17 \\
\hline Pectoral fins length & PfL & $\mathrm{cm}$ & $1.16 \pm 0.02$ & 1.00 & 1.41 & 9.30 & 0.10 \\
\hline Ventral fins length & VfL & $\mathrm{cm}$ & $0.97 \pm 0.02$ & 0.75 & 1.20 & 12.40 & 0.12 \\
\hline Anal fin height & AfH & $\mathrm{cm}$ & $1.06 \pm 0.03$ & 0.83 & 1.52 & 15.22 & 0.16 \\
\hline Caudal fin length & $\mathrm{Cfl}$ & $\mathrm{cm}$ & $1.52 \pm 0.03$ & 1.27 & 1.96 & 10.02 & 0.15 \\
\hline
\end{tabular}

Note: MU - measurement unit; X - mean value; sx - standard error; V\% - coefficient of variation; s - standard deviation; Min - minimum value; Max - maximum value. 
Tab. 6. Body proportions for specimens collected from the 4 locations

\begin{tabular}{ccccc}
\hline LOCATION & Crisul Repede & Somesul Mic & Aries & Viseu \\
\cline { 1 - 4 } Ratio & $19.75 \%$ & $18.89 \%$ & $18.15 \%$ & $18.81 \%$ \\
\hline HL/TL & $21.28 \%$ & $21.48 \%$ & $19.52 \%$ & $19.18 \%$ \\
\hline H/TL & $7.94 \%$ & $7.76 \%$ & $7.53 \%$ & $7.42 \%$ \\
\hline h/TL & $18.43 \%$ & $18.22 \%$ & $19.52 \%$ & $18.44 \%$ \\
\hline DfH/TL & $28.86 \%$ & $30.35 \%$ & $28.93 \%$ & $33.55 \%$ \\
\hline SnL/HL & $100 \%$ & $101.92 \%$ & $105.76 \%$ & $105.76 \%$ \\
\hline LjL/UjL & $28.35 \%$ & $29.16 \%$ & $32.07 \%$ & $30.92 \%$ \\
\hline ED/HL & & & & \\
\hline
\end{tabular}

specimens. The maximum height in total length ratio is above $21 \%$ for Crisul Repede and Somesul Mic specimens, while it is slightly over $19 \%$ for Aries and Viseu spirlin. The minimum height in total length ratio is situated between $7-9 \%$ for all locations. The dorsal fin height in total length ratio is found between $18-19 \%$ for spirlin from Crisul Repede, Somesul Mic and Viseu, but it is well over $19 \%$ for Aries River. The snout length in head length ratio is above $28 \%$ for Crisul Repede and Aries specimens, over 30\% for Somesul Mic specimens and over $33 \%$ for Viseu specimens. The lower jaw length is equal to the upper jaw length for specimens from Crisul Repede, but as far as the other 3 sampling points are concerned, the lower jaw length exceeds the upper jaw length. The eye diameter in head length ratio also differs in the sampling points, being over $28 \%$ for Crisul Repede specimens, over 29\% for Somesul Mic specimens, over $30 \%$ for Viseu specimens and over $32 \%$ for Aries River spirlin.

\section{CONCLUSION}

Depending on the habitat in which spirlin is found, morphometric plasticity may be present. Such is the case of spirlin found in the main four catchments of Transylvania: Somes catchment, Mures catchment, Cris catchment and Viseu catchment. The studied specimens of spirlin presented notable differences in body part ratios, being adapted to different environment conditions.

\section{REFERENCES}

1. AziziF,AnvarifarH,Mousavi-SabetH(2015).Morphological Differentiation Between Isolated Populations of Caspian spirlin (Alburnoides eichwaldii) (Pisces: Cyprinidae) Affected by Dam. International Journal of Animal Biology, Vol. 1, No. 2, pp. 28-37.
2. Bartholomew A, Bohnsack JA (2005). A review of catchand-release angling mortality with implications for notake reserves. Fish Biology and Fisheries, 15: pp. 129-154.

3. Bănăduc D, Stroilă V, Curtean-Bănăduc A (2013). The fish fauna of the Timiş river (Banat, Romania). Transylv. Rev. Syst. Ecol. Res. 15 - special issue "The Timiş River Basin".

4. Bănărescu P (1964). Fauna Republicii Populare Romîne, Pisces-Osteichthyes (Peşti ganoizi şi osoşi), Vol. XIII. Ed. Academiei Republicii Populare Române, Bucureşti.

5. Berg LS (1964). Freshwater fi shes of the U.S.S.R. and adjacent countries. Volume II, 4th Edition, Israel Program for Scientifi c Translations Ltd. (Russian version published 1949).

6. Breitenstein ME, Kirchhofer A (2000). Growth, age structure and species association of the cyprinid Alburnoides bipunctatus in the River Aare, Switzerland. Folia Zoologica 49, 59-68.

7. Cocan D, Popescu F, Laţiu C, Coşier V, Coroian A, Negrea O, Ihuț A, Mireşan V (2015). Meristic and Morphometric Characteristics of Spirlin, Alburnoides bipunctatus Bloch 1782 (Actinopterygii: Cyprinidae) of the Letca Area - Someş River. Bulletin UASVM Animal Science and Biotechnologies 72(2).

8. Cooke SJ, Schramm HL (2007). Catch-and-release science and its application to conservation and management of recreational fisheries. Fisheries Management and Ecology, 14, pp. 73-79.

9. Copp GH, Kováč V, Siryová S (2010). Microhabitat use by stream-dwelling spirlin Alburnoides bipunctatus and accompanying species: implications for conservation. Folia Zoologica, 59 (3): 240-256.

10. Curtean-Bănăduc A, Bănăduc D (2007). Benthic macroinvertebrate and fish communities of some southern Târnava Mare river tributaries (Transylvania, Romania). Transylv. Rev. Syst. Ecol. Res. 4, "The Saxon Villages Region of southeast Transylvania".

11. Györe K, Józsa V, Gál D, Lengyel P (2013). Fish faunal studies in the Körös river system. AACL Bioflux 6(1):3441.

12. Hensel K, Mužík V (2001). Červený (ekosozologický) zoznam mihúl' (Petromyzontes) a rýb (Osteichthyes) Slovenska [Red (ecosozological) list of lampreys (Petromyzontes) and fishes (Osteichthyes) of Slovakia]. In: Baláž D., Marhold K. \& Urban P. (eds), Červený zoznam 
rastlín a živočíchov Slovenska. Ochr. Prír. 20 (Suppl.): 143-145 (in Slovak).

13. Jurajda P, Adámek Z, Janáč M, Valová Z (2007). Fish and macrozoobenthos in the Vlára stream drainage area (Bílé Karpaty Mountains). Czech J. Anim. Sci. 52: 214-225.

14. Jurajda P, Hohausova E, Prasek V, Dvorak M (1996). Fishes of Polana Mountain Streams. Biologia, 51:173-178.

15. Kováč V, Katin S, Copp GH, Siryová S (2006). Ontogenetic variability in external morphology and microhabitat use of spirlin Alburnoides bipunctatus from the River Rudava (Danube catchment). Journal of Fish Biology, 68, 12571270.

16. Ladiges W, Vogt D (1979). Die Su3wasserfische Europas. Paul Parey, Hamburg \& Berlin, 299 pp.

17. Lelek A (1987). The freshwater fishes of Europe, Vol. 9 , Threatened Fishes of Europe. Aula-Verlag, Wiesbaden. $343 \mathrm{pp}$.

18. Lusk S, Lusková V, Halačka K, Šlechta V, Šlechtová V (1998). Trends and production of fish communities of the barbel zone in a stream of the Czech Republic. Folia Zool 47 (Suppl. 1): 67-72.
19. Papadopol M, Cristofor S (1980). Recherches sur L'ecologie de deux populations Alburnoides b. bipunctatus (Bloch), des eaux de la Roumanie (Pisces, Cyprinidae). - Trov. Mus. Hist.nat. Grigore Antipa, 22: 483-493.

20. Peňáz M (1995): Alburnoides bipunctatus. In: Baruš V. \& Oliva O. (eds.), Fauna ČR a SR/Mihulovci a ryby II [Fauna of the Czech Republic and Slovakia/Lampreys and fishes II]. Academia, Prague: 151-154. (in Czech).

21. Swain DP, Ridell BE, Murray CB (1991). Morphological differences between hatchery and wild populations of coho salmon (Oncorhynchus kisutch): environmental versus genetic origin. CAN J FISH AQUAT SCI, 48, 17831791.

22. Tales E, Keith P, Oberdorff T (2004). Density-range size relationships in French riverine fishes. Oecologia 138: 360-370.

23. Treer T, Piria M, Aničić I, Safner R, Tomljanović T (2006). Diet and growth of spirlin, Alburnoides bipunctatus in the barbel zone of the Sava River. Folia Zoologica, 55(1): 97-106. 